ARTICLE

https://doi.org/10.1038/s41467-019-08843-5

\title{
Tailoring vertical phase distribution of quasi-two- dimensional perovskite films via surface modification of hole-transporting layer
}

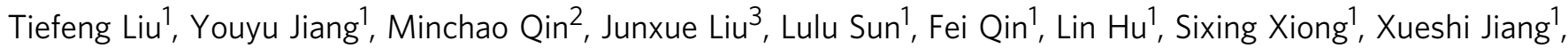
Fangyuan Jiang ${ }^{1}$, Ping Peng ${ }^{4}$, Shengye $J_{i n}^{3}$, Xinhui Lu $^{2} \&$ Yinhua Zhou (D ${ }^{1}$

Vertical phase distribution plays an important role in the quasi-two-dimensional perovskite solar cells. So far, the driving force and how to tailor the vertical distribution of layer numbers have been not discussed. In this work, we report that the vertical distribution of layer numbers in the quasi-two-dimensional perovskite films deposited on a hole-transporting layer is different from that on glass substrate. The vertical distribution could be explained by the sedimentation equilibrium because of the colloidal feature of the perovskite precursors. Acid addition will change the precursors from colloid to solution that therefore changes the vertical distribution. A self-assembly layer is used to modify the acidic surface property of the hole-transporting layer that induces the appearance of desired vertical distribution for charge transport. The quasi-two-dimensional perovskite cells with the surface modification display a higher open-circuit voltage and a higher efficiency comparing to reference quasi-twodimensional cells.

\footnotetext{
${ }^{1}$ Wuhan National Laboratory for Optoelectronics, and School of Optical and Electronic Information, Huazhong University of Science and Technology, Wuhan 430074, China. ${ }^{2}$ Department of Physics, The Chinese University of Hong Kong, New Territories, Hong Kong 999077, China. ${ }^{3}$ State Key Laboratory of Molecular Reaction Dynamics and Collaborative Innovation Center of Chemistry for Energy Materials (iChEM), Dalian Institute of Chemical Physics, Chinese Academy of Sciences, 457 Zhongshan Road, Dalian 116023, China. ${ }^{4}$ School of Materials Science and Engineering, Huazhong University of Science and Technology, Wuhan 430074, China. These authors contributed equally: Tiefeng Liu, Youyu Jiang. Correspondence and requests for materials should be addressed to X.L. (email: xinhui.lu@cuhk.edu.hk) or to Y.Z. (email: yh_zhou@hust.edu.cn)
} 
T hree-dimensional (3D) organic-inorganic hybrid perovskites have drawn enormous research interest in the past few years for photovoltaic applications due to their unique optoelectronic properties, such as high absorption coefficient ${ }^{1}$, small exciton binding energy ${ }^{2}$, long carrier diffusion lengths $s^{3,4}$, excellent charge mobility ${ }^{5}$, low defect densities ${ }^{6,7}$, and tunable bandgaps ${ }^{8,9}$. The highest power conversion efficiency (PCE) of 3D perovskite solar cells has reached $23.7 \%{ }^{10}$. However, the chemical activity and instability of perovskite ${ }^{11-14}$, especially over moisture ${ }^{15,16}$, restrict its development and applications.

In recent years, the layered quasi-two-dimensional (Q-2D) $\left(\mathrm{A}_{1}\right)_{2}\left(\mathrm{~A}_{2}\right)_{n-1} \mathrm{~B}_{n} \mathrm{X}_{3 n+1}$ [where $\mathrm{A}_{1}$ is large-size bulky organic cation, such as butylammonium (BA) or phenethylammonium (PEA); $\mathrm{A}_{2}$ is $\mathrm{Cs}$, methylammonium (MA) or formamidinium (FA); $\mathrm{B}$ is $\mathrm{Pb}$ or $\mathrm{Sn}$; $\mathrm{X}$ is halide anion; and the integer $n$ is number of layered inorganic $\left[\mathrm{PbI}_{6}\right]$ sheets between two bulky organic cation spacers] perovskites have shown potential to improve the physico-chemical stability ${ }^{17-20}$. The hydrophobic feature of longer alkyl chain in bulky organic cation ${ }^{21-23}$ and the strong van der Waals interaction between the capping organic molecules and the $\left[\mathrm{PbI}_{6}\right]$ unit $^{24}$ contributes to the enhancement of stability. The large-sized organic cations in the Q-2D structure isolate the electronic coupling between the $n$-layer $\left[\mathrm{PbI}_{6}\right]$ sheets, resulting in the formation of the multi-quantum-wells in the Q-2D films. Photogenerated excitons are confined within the layered crystalline lattice in the multi-quantum-wells which leads to larger exciton binding energy than that of their $3 \mathrm{D}$ counterparts ${ }^{25,26}$. The insulating organic spacers will also inhibit the out-of-plane charge transport ${ }^{27,28}$. To ensure the efficient collection of photo-generated charge carriers, hot-casting deposition approach ${ }^{29}$, solvent engineering ${ }^{30-32}$, additive engineering $\left(\mathrm{SCN}^{-}, \mathrm{MACl}\right)^{33-35}$, and composition optimization (FA, Cs) ${ }^{36,37}$ have been adopted to facilitate the vertical growth. Currently, the PCE for small- $n$ Q-2D $(n=4)$ perovskite solar cells has reached $12.81 \%$ (inverted structure) ${ }^{36}$ and $13.68 \%$ (conventional structure) ${ }^{37}$. It is still lower than that of $3 \mathrm{D}$ perovskite solar cells.

Different from their 3D counterparts, previous reports have shown that the fabricated Q-2D perovskite films are actually a mixture of multiple perovskite phases with different $n$ values instead of a homogeneous perovskite phase with an identical $n$ value. These multiple phases are arranged spontaneously along the direction perpendicular to the substrate from small- $n$ to large- $n$ (from substrate side to the top surface of films $)^{38,39}$. Jin and co-workers have demonstrated that such multiple-phase structure in Q-2D perovskite films contain desired energy band alignment, which benefits the electron-hole separation and facilitates the charge transfer between the phases with small $n$ and large- $n$. The vertical energy alignment from small- $n$ to large- $n$ is shown in the Supplementary Figure 1 (note: the energy levels of the Q-2D perovskite with different $n$ are extracted from the work reported by Kanatzidis ${ }^{21}$ where the energy level measurements are performed on homologous $2 \mathrm{D}$ perovskite crystals). The internal charge transfer property facilitates the charge extraction to the electrodes for photovoltaic applications in inverted device structure ${ }^{38,40,41}$.

In this work, we find that the desired vertical phase distribution of $n$ from the small to the large disappears when the Q-2D perovskite is deposited on the hole-transporting layer (HTL) of poly(3,4ethylenedioxythiophene): poly(styrene sulfonate) (PEDOT:PSS), which is different from that on the glass substrate. We employ a selfassembly layer of 4-bromobenzenediazonium tetrafluoroborate covalently anchored onto the surface of the PEDOT:PSS that can induce the appearance of the desired vertical phase distribution of $n$ in the Q-2D perovskite films. The PEDOT:PSS modified by 4bromobenzenediazonium tetrafluoroborate is henceforth referred to as BrB-PEDOT:PSS. The devices with BrB-PEDOT:PSS display reduced non-radiative recombination and higher $V_{\mathrm{OC}}(1.11 \mathrm{~V})$ comparing to the reference Q-2D perovskite cells without the surface anchoring layer. The $(\mathrm{BA})_{2}\left(\mathrm{MA}_{0.95} \mathrm{Cs}_{0.05}\right)_{3} \mathrm{~Pb}_{4} \mathrm{I}_{13}$ Q-2D cells with the BrB-PEDOT:PSS HTL show a PCE of $13.74 \%$.

\section{Results}

Observation of vertical phase distribution and tailoring. We began with the study on the vertical phase distribution of Q-2D perovskite on glass substrate using photoluminescence (PL) spectroscopy. BA is used for the large-size cations to generate Q$2 \mathrm{D}$ perovskite layers. To enhance the films quality, $5 \% \mathrm{Cs}$ was added into the Q-2D perovskite precursors ${ }^{37}$. The stoichiometry of precursor determines the chemical structure of Q-2D perovskite is $(\mathrm{BA})_{2}\left(\mathrm{MA}_{0.95} \mathrm{Cs}_{0.05}\right)_{3} \mathrm{~Pb}_{4} \mathrm{I}_{13}$. PL measurements were excited from both the front side (perovskite surface) as well as the back side (glass side). As shown in Fig. 1a, when excited from the front side, the PL spectrum shows a single emission peak at about $770 \mathrm{~nm}$ (the insert of Fig. 1a). When excited from the back side, multiple emission peaks at higher energy appear. Such difference observed in the PL spectrum is consistent with the previous reports ${ }^{38,39}$. It suggests that there are small- $n$ phases at the bottom of the perovskite films near the substrate and large- $n$ phases at the upper surface of the perovskite film. This vertical phase distribution is favorable for charge separation and transport in inverted perovskite solar cell structure ${ }^{35,38,39}$.

Then, we tried to build this vertical phase distribution on PEDOT:PSS films that are typically used as HTL in the inverted Q-2D perovskite solar cells. However, it is surprising that the multiple emission peaks associated with small- $n$ phases at the bottom side nearly disappear (Fig. 1a) when the Q-2D perovskite films are grown on PEDOT:PSS layer with the same film casting method. The PEDOT:PSS suppresses the vertical phase distribution of the Q-2D perovskite.

To obtain the desired vertical phase distribution on PEDOT: PSS HTL, we use a small molecule of 4-bromobenzenediazonium tetrafluoroborate to react with PEDOT:PSS (BrB-PEDOT:PSS) to tune the surface properties of the PEDOT:PSS. Through the interaction between the diazo cation and $-\mathrm{SO}_{3}{ }^{-}$group followed by post-annealing, the ionic bond is converted to covalent bond (Fig. 1c). This covalent modification was verified using the Fourier transform infrared (FT-IR) spectrum (Supplementary Figure 2). The symmetric stretching band of $\mathrm{O}=\mathrm{S}=\mathrm{O}$ changes from 1176 (in PEDOT:PSS) to $1172 \mathrm{~cm}^{-1}$.(in BrB-PEDOT:PSS). That suggests there exists the conversion of $-\mathrm{SO}_{3}{ }^{-}$(ionic bond) to $-\mathrm{SO}_{2}-\mathrm{O}-$ (covalent bond ${ }^{42,43}$. After modification, the zeta potential of the PEDOT:PSS shifts from -53 to $-29 \mathrm{mV}$ (Fig. 1b) and the contact angle increases from $18.62^{\circ}$ to $46.79^{\circ}$ (Fig. 1d, e). The shift of the zeta potential also confirms the conversion of ionic bond to covalent bond where less negative ions exist after the reaction. The larger contact angle further confirms the modification. Importantly, the small- $n$ peaks of Q-2D perovskite films deposited on BrB-PEDOT:PSS become more pronounced compared to that on PEDOT:PSS (Fig. 1a). The Q-2D perovskite films on both HTLs have similar absorption spectrum (Supplementary Figure 3).

To further investigate the compositional gradient $\mathrm{BA}$ and MA in the Q-2D perovskite films, we used depth-profiling Xray photoelectron spectroscopy (XPS) to detect the $\mathrm{C} / \mathrm{Pb}$ atom ratio where the number of $\mathrm{C}$ atom in $\mathrm{BA}$ is larger than that in $\mathrm{MA}$. As etching time increases, the $\mathrm{C} / \mathrm{Pb}$ ratio becomes larger when the substrate is BrB-PEDOT:PSS, as shown in Fig. 1f, which indicates that the Q-2D perovskite films fabricated on the BrB-PEDOT:PSS have more BA located at the bottom of perovskite film (close to the substrate side) compared to those fabricated on the PEDOT:PSS. That is consistent with the results of PL spectra. 

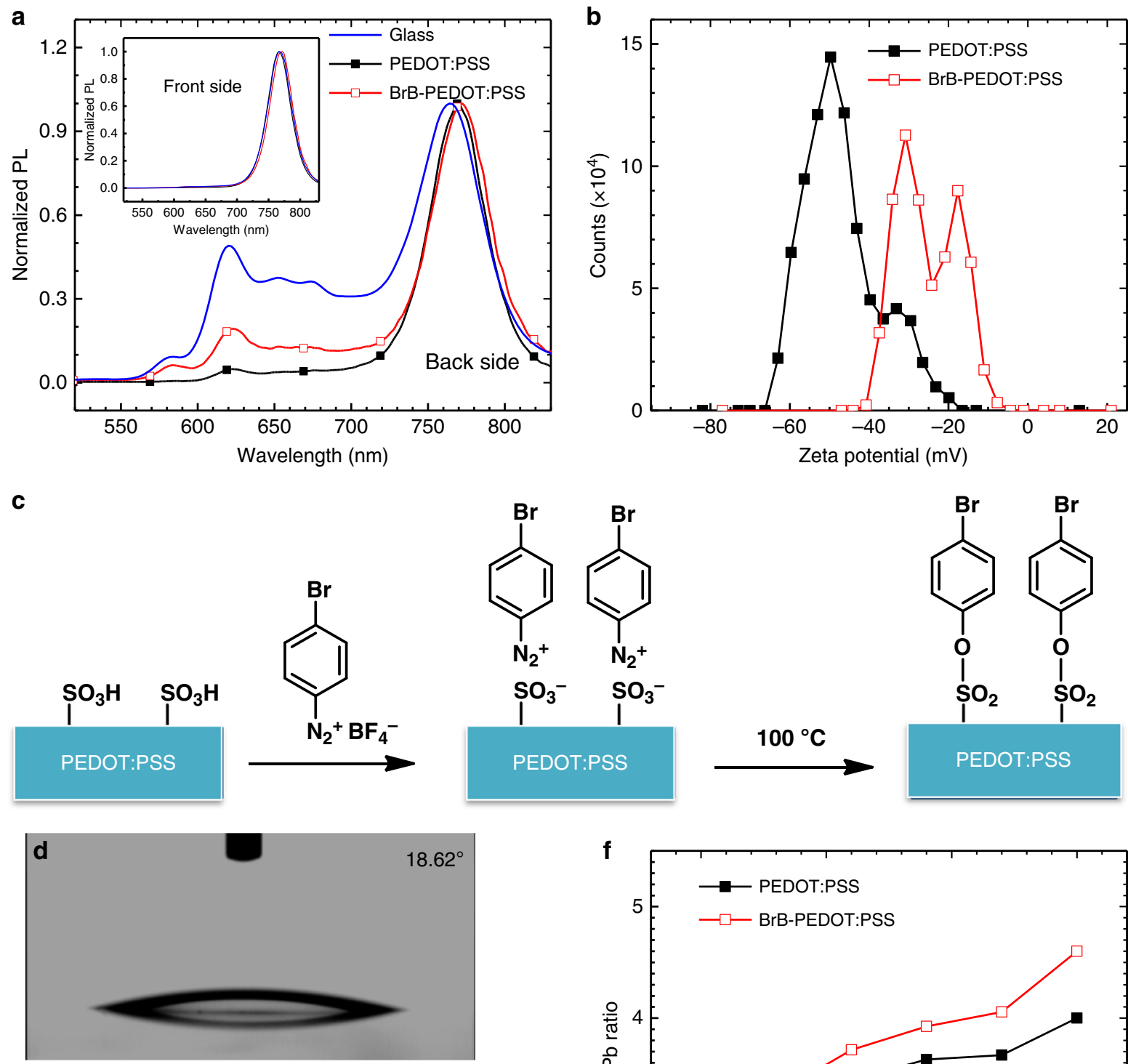

PEDOT:PSS
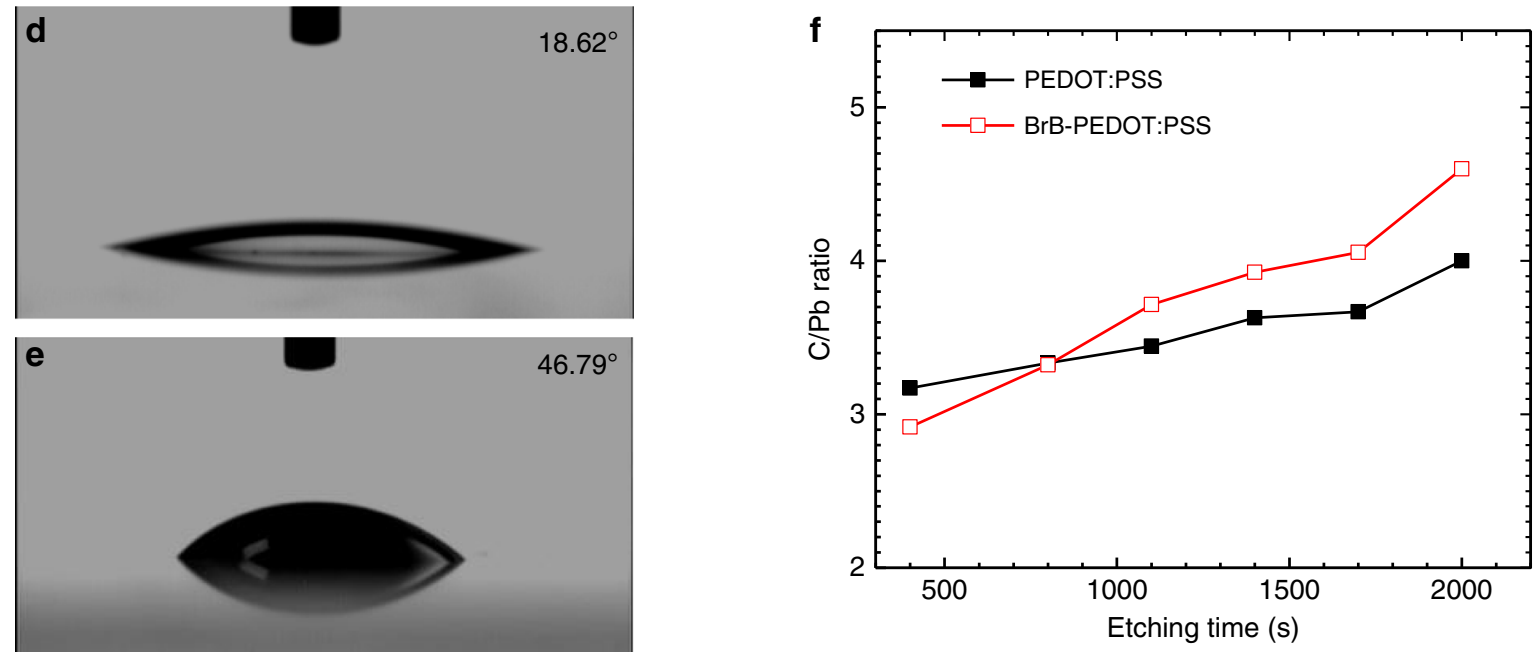

Fig. 1 Observation of vertical phase distribution and tailoring in Q-2D perovskite films. a PL spectra of Q-2D perovskite films on different substrates excited from the back side (substrate side). The inset is the PL spectra of Q-2D perovskite films on different substrates excited from the front side; $\mathbf{b}$ the zeta potential of PEDOT:PSS and BrB-PEDOT:PSS; $\mathbf{c}$ the schematic of covalent modification on PEDOT:PSS via a molecule of 4-bromobenzenediazonium tetrafluoroborate. Contact angle images of water on: $\mathbf{d}$ PEDOT:PSS and $\mathbf{e}$ BrB-PEDOT:PSS. $\mathbf{f X P S}$ depth profiles of $\mathrm{C} / \mathrm{Pb}$ atom ratios as a function of etching time for the Q-2D films. (etching direction: surface of the perovskite film to the substrate side)

Understanding the origin of vertical phase distribution. The vertical phase distribution with different $n$ values is dependent on the distribution of large-size capping cation of BA in the Q-2D perovskite films. In previous studies, the perovskite precursors have been reported in the form of colloids ${ }^{44,45}$. Here, the Tyndall effect of Q-2D perovskite precursor is shown in Fig. $2 \mathrm{~b}$ that confirms the colloidal feature of the precursor. The vertical distribution of the colloids can be analyzed with the equation of sedimentation equilibrium: ${ }^{46}$

$$
\frac{c_{2}}{c_{1}}=\mathrm{e}^{-\frac{\mathrm{N}_{\mathrm{A}} v\left(\rho-\rho_{0}\right)\left(H_{1}-H_{2}\right) g}{R T}}
$$

where the $c_{1}$ and $c_{2}$ are the colloidal concentration at the height of $H_{1}$ and $H_{2}$; the $\mathrm{N}_{\mathrm{A}}$ is the Avogadro constant; the $v$ is the volume of the colloidal particle; the $\rho$ and $\rho_{0}$ denote the density of the 
a

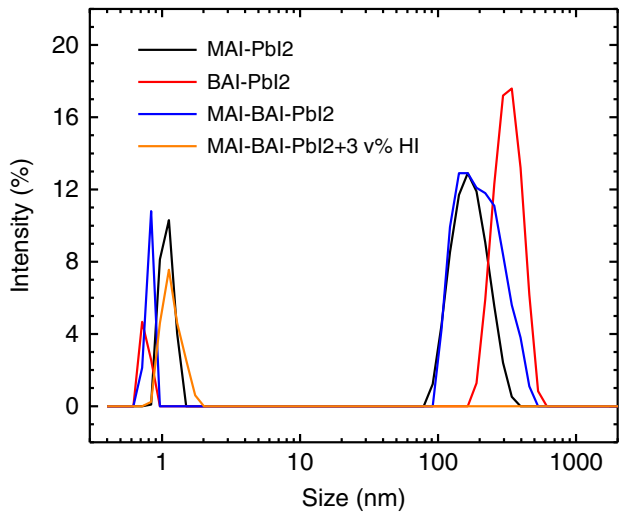

d

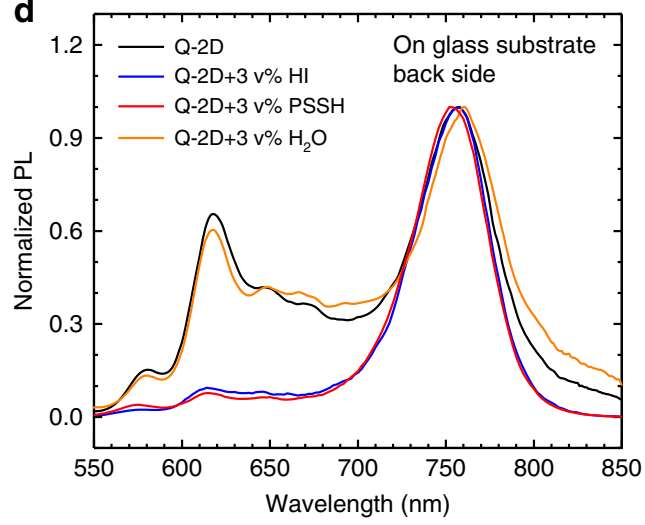

b

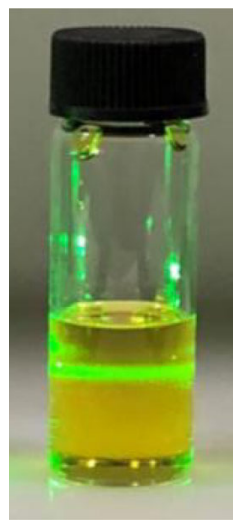

Without $\mathrm{HI}$ addition

e

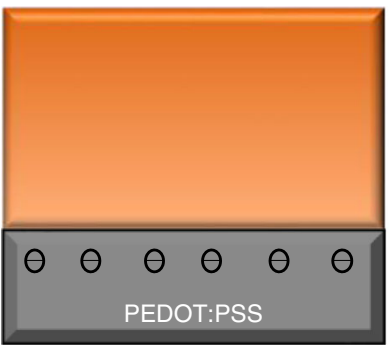

C

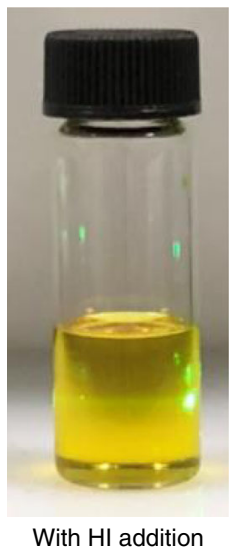

With $\mathrm{HI}$ addition

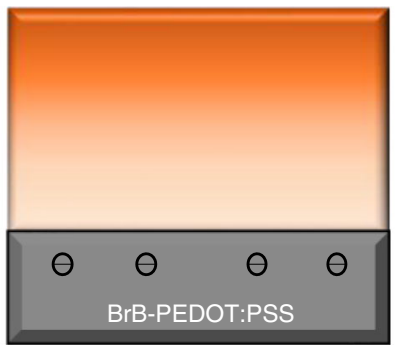

Fig. 2 Origin of vertical phase distribution in the Q-2D perovskite films. a Colloid size distribution of perovskite precursors in GBL:DMSO mixed solvents. The mole ratio of MAI- $\mathrm{Pbl}_{2}$ is 1:1, presenting the target $n$ is infinity. The mole ratio of $\mathrm{BAI}-\mathrm{Pbl}_{2}$ is 2:1, presenting the target $n$ is 1 . The mole ratio of MAI-BAI$\mathrm{Pbl}_{2}$ is $3: 2: 4$, presenting the target $n$ is 4 . MAI-BAI-Pbl $2+3 \mathrm{v} \% \mathrm{HI}$ denotes 3 vol.\% $\mathrm{HI}$ is added into the precursor of $\mathrm{MAl}-\mathrm{BAI}-\mathrm{Pbl}{ }_{2}$ (3:2:4). Tydall effect photographs of the Q-2D perovskite precursors: $\mathbf{b}$ without or $\mathbf{c}$ with 3 vol.\% HI addition. $\mathbf{d} \mathrm{PL}$ spectra of Q-2D perovskite films (with or without acid addition) deposited on glass substrates excited from back side. e Schematic of phase distribution (small- $n$ to large- $n$ phase from substrate side to the top side) in Q-2D perovskite films on PEDOT:PSS and BrB-PEDOT:PSS substrates

colloidal particles and the solvent, respectively; the $\mathrm{g}$ is gravitational acceleration constant; the $\mathrm{R}$ is constant and the $T$ is the temperature.

Figure 2a shows the dynamic light scattering (DLS) results of $\mathrm{MAI}_{\mathrm{x}}-\mathrm{BAI}_{\mathrm{y}}-\mathrm{PbI}_{2}$ precursors in the solvents. We take the MAI$\mathrm{PbI}_{2}$ (the mole ratio is $1: 1$, presenting the $n$ is infinity) and BAI$\mathrm{PbI}_{2}$ (the mole ratio is $2: 1$, presenting the $n$ is 1 ) as the example. As shown in Fig. 2a, the average colloidal sizes of the MAI-PbI clusters and $\mathrm{BAI}-\mathrm{PbI}_{2}$ cluster are about $180 \mathrm{~nm}$ and $320 \mathrm{~nm}$, respectively. Thus, at a certain height of $H_{1}$ and $H_{2}$ (where $H_{1}>$ $\mathrm{H}_{2}$, and $\mathrm{H}_{2}$ is near the substrate), since BAI-PbI 2 colloid has a larger volume than MAI- $\mathrm{PbI}_{2}$ colloid $\left(v_{\mathrm{BA}}>v_{\mathrm{MA}}\right.$,), the difference in BAI- $\mathrm{PbI}_{2}$ concentration at the height of $H_{2}$ and $H_{1}$ [denoted as $\left(\frac{c_{2}}{c_{1}}\right)_{\mathrm{BA}}$ ] and that of $\mathrm{MAI}_{-} \mathrm{PbI}_{2}$ [denoted as $\left(\frac{c_{2}}{c_{1}}\right)_{\mathrm{MA}}$ ] will meet the equation: $\left(\frac{c_{2}}{c_{1}}\right)_{\mathrm{BA}}>\left(\frac{c_{2}}{c_{1}}\right)_{\mathrm{MA}}$, which can be transformed into $\frac{\left(c_{2}\right)_{\mathrm{BA}}}{\left(c_{2}\right)_{\mathrm{MA}}}>\frac{\left(c_{1}\right)_{\mathrm{BA}}}{\left(c_{1}\right)_{\mathrm{MA}}}$. Therefore, the mole ratio of BAI/MAI at the bottom is larger than that at the top. This explains the phenomenon generally observed in Q-2D perovskite films that small- $n$ phases locate at the bottom of the perovskite films while large- $n$ phases locate at the upper surface of the perovskite films when deposited on glass substrates.

However, when deposited on the PEDOT:PSS, the vertical distribution of $n$ from the small (bottom side) to the large (top side) disappears in the Q-2D perovskite films. The rule of sedimentation equilibrium can't explain the observation of the
Q-2D perovskite films deposited on the PEDOT:PSS. First we consider the possible effect of surface energy. Contact angle on glass substrate $\left(43.48^{\circ}\right.$, Supplementary Figure 4$)$ which is similar to that of BrB-PEDOT:PSS $\left(46.79^{\circ}\right)$. Considering that their PL spectra are quite different, the surface energy is not the main reason of the vertical $n$ distribution. Then, we hypothesize the change of the vertical distribution of $n$ is associated to the change of the precursor solutions. The PSSH acid is soluble in the processing solvent of the perovskite (DMSO $)^{47,48}$. As shown in the Supplementary Figure 5, the XPS spectra show the removal of PSSH. After the spin-coating of pure DMSO on PEDOT:PSS, the intensity of the sulfur atoms in PSSH (binding energy between 166 and $172 \mathrm{eV}$ ) decreases, while the intensity of the sulfur atoms in PEDOT is unchanged (binging energy between 162 and $166 \mathrm{eV}$ ). Previously, it has been reported that the acid addition will reduce the colloid concentration in the perovskite precursors ${ }^{49-51}$. Because the PSSH itself is in colloid formation (the Tyndall effect of PSSH solution is shown in Supplementary Figure 6), we added $\mathrm{HI}$ instead of PSSH to study the effect. As shown in Fig. 2a, upon the addition of HI (3 vol.\%), the large-sized colloids disappear and the precursor becomes solution, which is accordance with the previous study 49,50 . The photographs of the Q-2D perovskite precursor with and without acid addition were shown in Fig. 2b, c, respectively. After the addition of the acid, light scattering can hardly be observed (Fig. 2c). To certify that the acid addition suppresses the vertical phase distribution, we deposited the Q-2D 
perovskite precursor with the addition of HI, PSSH, and water on glass substrates and measured their PL from the back side (glass side). As shown in Fig. 2d, the peaks of small- $n$ phases are much less pronounced when the HI or PSSH acid is added into the precursor.

Furthermore, we deposited Q-2D perovskite films on PEDOT: PSS, PSSH and DMSO treated PEDOT:PSS and measured their PL from the back side. The DMSO treatment denotes the spin coating of pure DMSO on top of the PEDOT:PSS film. As shown in Supplementary Figure 7, the peaks of small- $n$ phases are stronger on DMSO treated PEDOT:PSS surface than the films on the other two surface because of the fewer PSSH. After BrBmodification, the PSSH was converted into PSS-BrB and the ionic bond was converted into covalent bond. The non-acid PEDOT: PSS surface after $\mathrm{BrB}$ modification will maintain the colloidal property of the precursors. Therefore, the vertical distribution of $n$ can be obtained when the Q-2D perovskite is deposited on the BrB-PEDOT:PSS. Figure 2e demonstrates the schematic of phase distribution in Q-2D films deposited on PEDOT:PSS and BrBPEDOT:PSS substrates.

Morphology and crystallinity of the Q-2D perovskite films. We also characterize the morphology and the crystallinity with scanning electron microscopy (SEM) and grazing incidence wideangle X-ray scattering (GIWAXS) to investigate the perovskite films on different HTLs. From the SEM images shown in Fig. 3a, $b$, both the films show the uniform and compact coverage. Besides, both the GIWAXS patterns demonstrate discrete Bragg spots, indicating highly orientated crystalline (shown in Fig. 3c, d). Moreover, the orientated structure is beneficial for efficient charge carrier transport ${ }^{29}$. The Q-2D perovskite films on BrBPEDOT:PSS show the same set of diffraction spots as that on PEDOT:PSS substrate, but stronger intensity. The GIWAXS intensity profiles integrated over a polar angle from 0 to 90 degree are shown in the Supplementary Figure 8. The higher crystallinity can be attributed to the more hydrophobic substrate of $\mathrm{BrB}$ PEDOT:PSS ${ }^{52}$.

Photovoltaic performance of devices. We fabricated photovoltaic devices in the planar invert structure of ITO/HTL/ (BA) ${ }_{2}\left(\mathrm{MA}_{0.95} \mathrm{Cs}_{0.05}\right)_{3} \mathrm{~Pb}_{4} \mathrm{I}_{13} / \mathrm{PCBM} / \mathrm{BCP} / \mathrm{Ag}$ (where the HTL is PEDOT:PSS or BrB-PEDOT:PSS) and measured devices under the AM 1.5G solar simulator. The device structure and the current density-voltage $(J-V)$ curves of devices are presented in Fig. 4a, b. Reference devices with PEDOT:PSS HTL delivered an champion PCE of $12.29 \%$ where open-circuit voltage $\left(V_{\mathrm{OC}}\right)$, short-circuit current density $\left(J_{\mathrm{SC}}\right)$, and fill factor $(\mathrm{FF})$ are $1.01 \mathrm{~V}$, $17.16 \mathrm{~mA} \mathrm{~cm}^{-2}$, and 0.71 , respectively. The performance is comparable to those reported in the literature of devices with the similar structure ${ }^{36}$. With the BrB-PEDOT:PSS as HTL, the PCE of the cells reaches $13.74 \%$ with an significant improvement of $V_{\mathrm{OC}}(1.11 \mathrm{~V})$. To the best of our knowledge, this is the highest $V_{\mathrm{OC}}$ regarding BA-based Q-2D $(n=4$, derived from the precursor formulation) based perovskite solar cell. Supplementary Table 1 summarized the previously reported BA-based Q-2D perovskite solar cell performance. The statistical data of 55 devices are summarized in Fig. $4 \mathrm{c}$ and Table 1 . The $V_{\mathrm{OC}}$ increases from $0.96 \pm 0.04$ to $1.08 \pm 0.02 \mathrm{~V}$ and the FF slightly increases from $0.68 \pm 0.02$ to $0.70 \pm 0.02$ while the $J_{\mathrm{SC}}$ nearly stays the same. Moreover, the BrB-PEDOT:PSS based devices have the narrower distribution of $V_{\mathrm{OC}}$. The $J_{\mathrm{SC}}$ was confirmed by the integration of the EQE spectrum (shown in Fig. 4d). The devices show negligible hysteresis under different scan directions and different scan rates (shown in Supplementary Figure 9 and 10). The stability efficiency at the maximum power point is shown in the
Supplementary Figure 11. Performance of devices processed from other conditions (using pure GBL solvent to process the perovskite layer, or the perovskite layer without Cs addition) is shown in Supplementary Figure 12 and 13, respectively. They both show inferior performance ( 8.6 and $10.9 \%$ after $\mathrm{BrB}$ modification, respectively) than the optimized devices with $5 \% \mathrm{Cs}$ addition and processed from GBL:DMSO mixed solvent (13.74\%).

It should be noted that we have also applied the BrB-PEDOT: PSS as HTL in conventional 3D perovskite solar cells. There is no such significant enhancement in $V_{\mathrm{OC}}$ (shown in Supplementary Figure 14). The $V_{\mathrm{OC}}$ is 0.93 and $0.91 \mathrm{~V}$ for the $3 \mathrm{D}$ perovskite solar cells with BrB-PEDOT:PSS and PEDOT:PSS HTLs, respectively. This also confirms the important role of the BrB-PEDOT:PSS tailoring the vertical phase distribution in Q-2D perovskite films. In 3D perovskite films, there is no such tailoring of $n$ values. The $n$ value is infinite throughout the entire $3 \mathrm{D}$ perovskite films.

Charge recombination and dynamics. The improvement of the solar cell performance after the BrB modification on PEDOT:PSS is mainly ascribed to the increase of $V_{\mathrm{OC}}$ from $0.96 \pm 0.04$ to 1.08 $\pm 0.02 \mathrm{~V}$. Based on the expression of $V_{\mathrm{OC}}=n_{\mathrm{if}} \mathrm{KT} / \mathrm{q} \ln \left(J_{\mathrm{SC}} / J_{\mathrm{sat}}\right)$ (where $\mathrm{K}$ is Boltzmann's constant, $T$ is the temperature, $n_{\mathrm{if}}$ is the diode ideality factor, $J_{\text {sat }}$ is the dark saturation current and $\mathrm{q}$ is elementary charge), smaller $J_{\text {sat }}$ that suggest lower recombination will result in higher $V_{\mathrm{OC}}{ }^{53,54}$. The $J_{\text {sat }}$ and $n_{\text {if }}$ values are extracted from dark $J-V$ characteristics fitted with one-diode equivalent circuit model (Fig. 5a). The resulting parameters are summarized in Supplementary Table 2. The $J_{\text {sat }}$ was fitted to $1.33 \times 10^{-9} \mathrm{~mA}$ $\mathrm{cm}^{-2}$ (cell with BrB-PEDOT) and $1.41 \times 10^{-7} \mathrm{~mA} \mathrm{~cm}^{-2}$ for PEDOT:PSS (cell with PEDOT:PSS), and the $n_{\text {if }}$ were fitted to 1.89 (cell with BrB-PEDOT) and 2.22 (cell with PEDOT:PSS). The calculated $V_{\mathrm{OC}}$ is $1.06 \mathrm{~V}$ for PEDOT:PSS based devices and $1.15 \mathrm{~V}$ for BrB-PEDOT:PSS based devices.

$V_{\mathrm{OC}}$ loss by non-radiative recombination $\left(\Delta V_{\mathrm{OC} \text {,non-rad }}\right)$ of perovskite solar cells is evaluated with the expression: $\Delta V_{\mathrm{OC}, \text { non }}$ $\mathrm{rad}=-\mathrm{KT} / \mathrm{q} \ln \left(\mathrm{EQE}_{\mathrm{EL}}\right)^{55-57}$, where $\mathrm{EQE} \mathrm{EL}_{\mathrm{EL}}$ is the electroluminescence (EL) EQE of cells working as light-emitting diodes. We measured the EL of the both cells with PEDOT:PSS and BrBPEDOT:PS HTL. When the injection current is equal to the $J_{\mathrm{SC}}$ at about $1.5 \mathrm{~V}$, the luminescent intensity is below the detection limit of the photodiode. At the applied voltage over $1.8 \mathrm{~V}$, the $\mathrm{EQE}_{\mathrm{EL}}$ of the cell with BrB-PEDOT:PSS is higher than that of the cell with PEDOT:PSS (Fig. 5b). The EL spectra of the two cells are shown in the Supplementary Figure 15 . The higher $\mathrm{EQE}_{\mathrm{EL}}$ suggests lower $V_{\mathrm{OC}}$ loss by the non-radiative recombination for the cell with BrB-HTL.

Trap density is important to the non-radiative recombination and the $V_{\mathrm{OC}}{ }^{58}$. Here, we evaluate the trap-state density using space charge limited current (SCLC) method by fabricating the hole-only devices (ITO/HTL/Q-2D/spiro-OMeTAD/Au, where the HTL is PEDOT:PSS or BrB-PEDOT:PSS). The trap-state density was calculated according to the equation:

$$
N_{\mathrm{t}}=\frac{2 \varepsilon_{\mathrm{o}} \varepsilon_{\mathrm{r}} V_{\mathrm{TFL}}}{\mathrm{q} L^{2}}
$$

where $\varepsilon_{\mathrm{o}}$ and $\varepsilon_{\mathrm{r}}$ are the dielectric constants of vacuum and the perovskite, $V_{\text {TFL }}$ is the threshold voltage of trap-filled limit (TFL) region, $\mathrm{q}$ is the elementary charge, and $L$ is the thickness of the film (about $400 \mathrm{~nm}$ ). From the current-voltage curves shown in Supplementary Figure 16a, we can calculate that the trap density of BrB-PEDOT:PSS based Q-2D films is about $(1.02 \pm 0.18) \times$ $10^{16} \mathrm{~cm}^{-3}$ which is lower than that of PEDOT:PSS based Q-2D films $\left((1.47 \pm 0.14) \times 10^{16} \mathrm{~cm}^{-3}\right)$. A slightly hysteresis can be observed in the SCLC measurement (Supplementary Figure 16b 
a

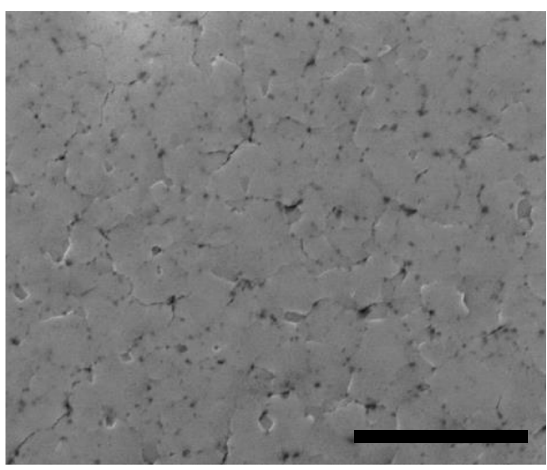

C

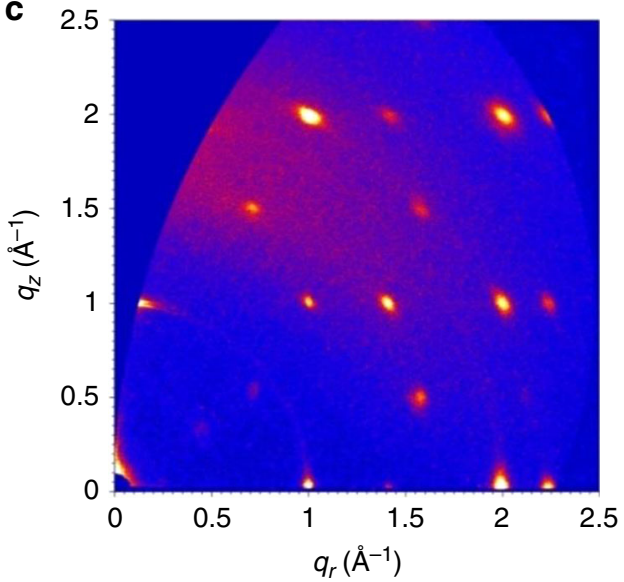

b

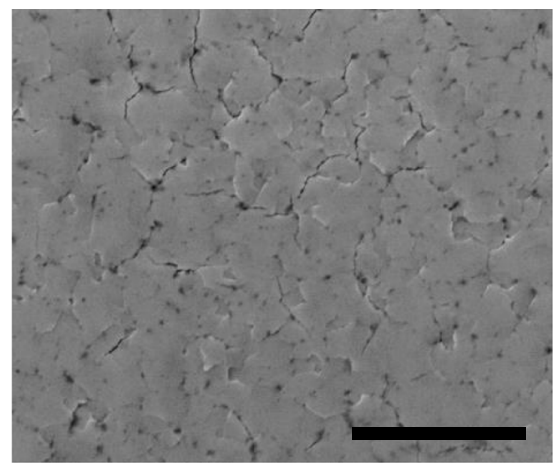

d

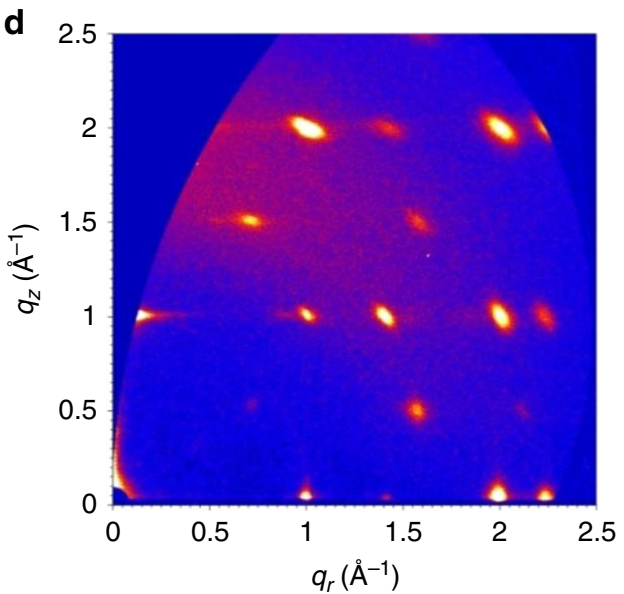

Fig. 3 Morphology and crystallinity of the Q-2D perovskite films. SEM images of Q-2D perovskite films on: a PEDOT:PSS and $\mathbf{b}$ BrB-PEDOT:PSS (the scale bar is $4 \mu \mathrm{m}$ ). GIWAXS scatting pattern of Q-2D perovskite films on: c PEDOT:PSS and $\mathbf{d}$ BrB-PEDOT:PSS

and $16 \mathrm{c}$ ), and the $V_{\mathrm{TFL}}$ values are similar in both scanning directions that derive similar trap densities.

To explore the influence of vertical phase distribution on the charge transport, ultrafast transient absorption (TA) measurement was performed to reveal the carrier dynamics. Supplementary Figure 17 shows the TA spectra at different delay time of the Q-2D perovskite films (about $400 \mathrm{~nm}$ thick) excited from the back side (glass side) at $480 \mathrm{~nm}, 1 \mu \mathrm{cm}^{-2}$ per pulse. The TA spectra show several bleach peaks which agree with the previous work $^{38,39}$. For BrB-PEDOT:PSS based Q-2D films, the bleach peak at $600 \mathrm{~nm}$ is stronger than that of PEDOT:PSS based Q-2D films. This also indicates more small- $n$ phases exist at the substrate side when the substrate is BrB-PEDOT:PSS. The bleach recovery of the small- $n$ phases and the generation of the large $n$ phase (at $740 \mathrm{~nm}$ ) are the proofs of electron transport from the small- $n$ to large- $n$ phases ${ }^{38}$. The TA kinetics probed at $740 \mathrm{~nm}$ is fitted as a function of time with bi-exponential function shown in Fig. 5c (fitting parameters are in Supplementary Table 3). In term of hole transfer, we measured the TA spectra on the Q-2D perovskite film excited from the front side (top side) at $740 \mathrm{~nm}$, $10 \mu \mathrm{J} \mathrm{cm}^{-2}$ per pulse. At this excitation wavelength, only the large- $n$ phase can be excited. However, besides the broad photoinduced absorption signal from large $n$, small- $n \quad(n=3$ perovskite phases) bleach peak is also observed (Supplementary Figure 18), owing to the hole transport from large- $n$ to small- $n$ phases. TA kinetics at $605 \mathrm{~nm}$ (Fig. 5d, after subtracting the photoinduced absorption contribution) is fitted to calculate the hole transport time from top to bottom (the fitting parameters are shown in Supplementary Table 4). When the substrate surface is BrB-PEDOT:PSS, both the electron and hole transfers in Q-2D perovskite films are faster compared to those in reference films on
PEDOT:PSS films (the rising part shown in Fig. $5 c$, d). The bleach recovery kinetics in Fig. $5 c$, d is attributed partially to the carrier extraction $^{59}$. The shorter delay time of the Q-2D perovskite films on BrB-PEDOT:PSS indicates that charge extraction occurs more efficiently in the case of BrB-PEDOT:PSS.

Supplementary Figure 19 shows the device performance evolution of the Q-2D perovskite solar cells stored in the dark in air without encapsulation. After kept in air for $1400 \mathrm{~h}$, both Q2D devices show a good stability. The PCEs remain $80 \%$ and $88 \%$ of their initial values for cells with PEDOT:PSS and BrB-PEDOT: PSS HTL, respectively. The main loss is due to the FF which might come from the degeneration between the interfaces of the Q-2D perovskite films and the electrodes. The better stability of the device with BrB-PEDOT:PSS HTL is probably attributed to the more hydrophobic property of BrB-PEDOT:PSS ${ }^{60}$.

\section{Discussion}

Vertical phase (layer numbers, $n$ ) distribution in Q-2D perovskite films has been observed ${ }^{38}$, but the driving force of vertical $n$ distribution has been not discussed or reported. In this work, we have reported that the vertical phase distribution of Q-2D perovskite films on glass substrates can be explained by the sedimentation equilibrium because of the colloidal feature of the precursors, and can be tailored by the surface modification and acid addition. Main observations and conclusions include: (1) the vertical phase distribution of Q-2D perovskite films on PEDOT:PSS HTL is different from the glass substrates. More small- $n$ phases in the Q-2D perovskite films form at the bottom side (close to substrate) when deposited on glass substrates, compared to the Q-2D perovskite films deposited on PEDOT:PSS layers; (2) The origin of the vertical 
a
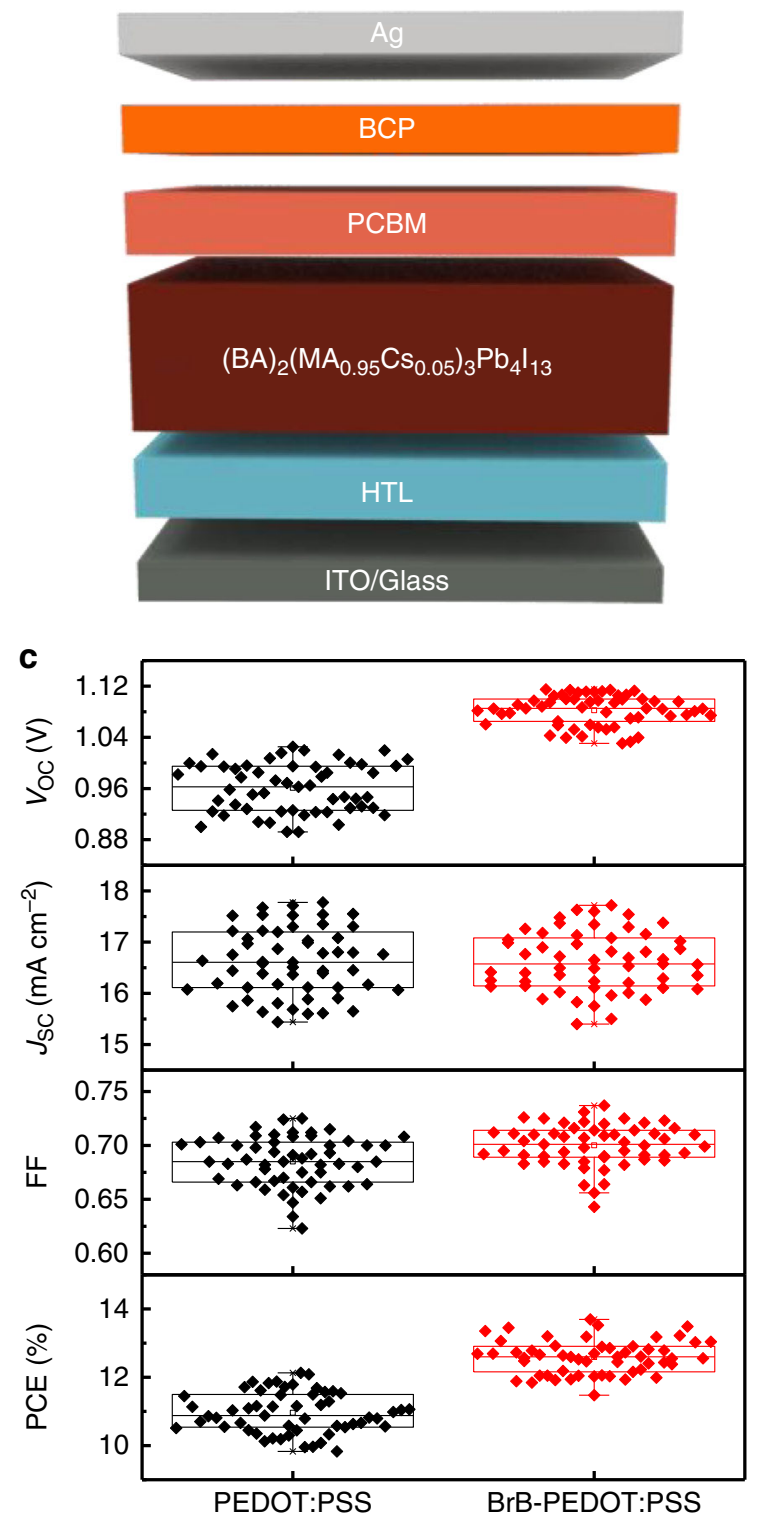

b

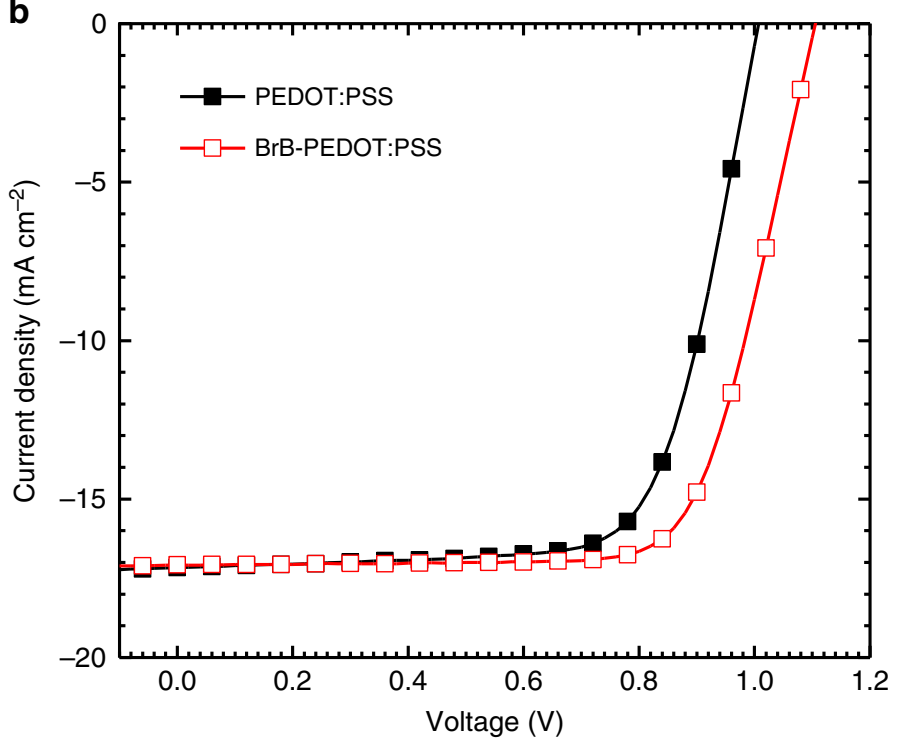

d

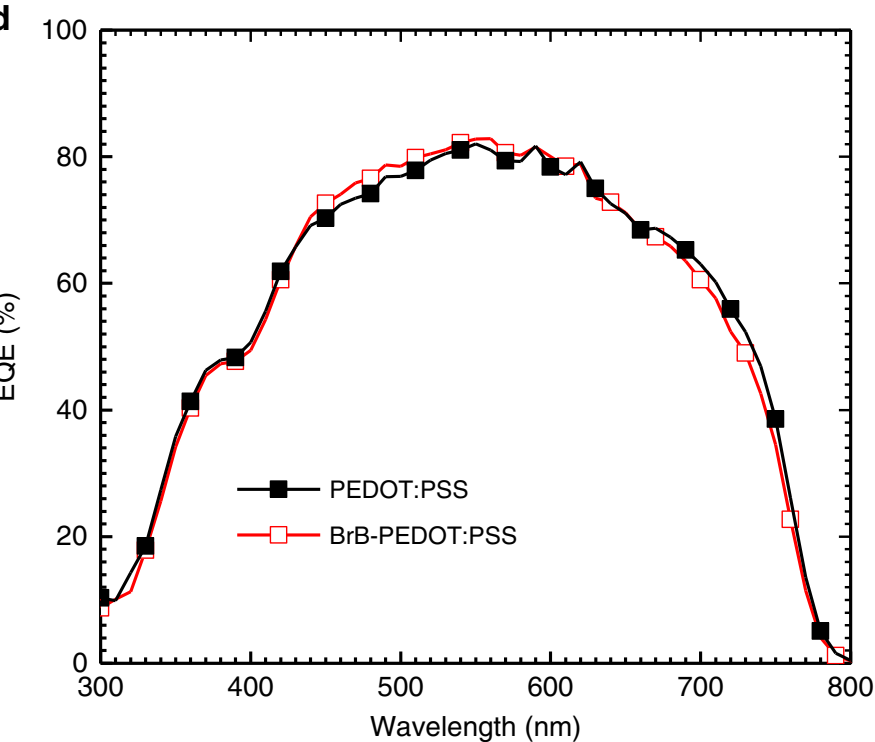

Fig. 4 Photovoltaic performance of devices with tailored vertical distribution. a Device structure of the Q-2D perovskite solar cells; $\mathbf{b} J-V$ characteristics of the cells with PEDOT:PSS and BrB-PEDOT:PSS; c Photovoltaic parameters of 55 devices for each HTL; (Boxes, square symbols, and horizontal bars indicate 25/75 percentile, mean, and min/max values, respectively). d EQE spectra of Q-2D perovskite solar cells with different PEDOT:PSS and BrB-PEDOT:PSS HTLS

Table 1 Photovoltaic parameters of Q-2D perovskite solar cells with PEDOT:PSS and BrB-PEDOT:PSS HTLs

\begin{tabular}{|c|c|c|c|c|c|c|}
\hline HTL & & $v_{\text {oc }}(\mathrm{V})$ & $J_{S C}\left(m A c m^{-2}\right)$ & $J_{S C, E Q E}\left(m A c m^{-2}\right)^{b}$ & FF (\%) & PCE (\%) \\
\hline \multirow[t]{2}{*}{ PEDOT:PSS } & Average & $0.96 \pm 0.04$ & $16.66 \pm 0.62$ & & $68.5 \pm 2.29$ & $10.96 \pm 0.60$ \\
\hline & Best $^{a}$ & 1.01 & 17.16 & 16.73 & 70.9 & 12.29 \\
\hline BrB-PEDOT:PSS & Average & $1.08 \pm 0.02$ & $16.62 \pm 0.57$ & & $70.0 \pm 1.92$ & $12.59 \pm 0.50$ \\
\hline
\end{tabular}

phase distribution of $n$ is the colloid property of the perovskite precursors (the colloid size on the order of $100-1000 \mathrm{~nm}$ ) that follows the sedimentation equilibrium. Once the colloid properties of precursors are changed to solution, the vertical distribution with different $n$ will not observed any more. The addition of the acid into the perovskite precursors will change the precursors from the colloid to solution; (3) For the surface of PEDOT:PSS, when the perovskite is deposited on top of it, the acid component of PSSH will be dissolved and changes colloid properties of the precursors into solution. The vertical distribution with different $n$ can hardly 
a

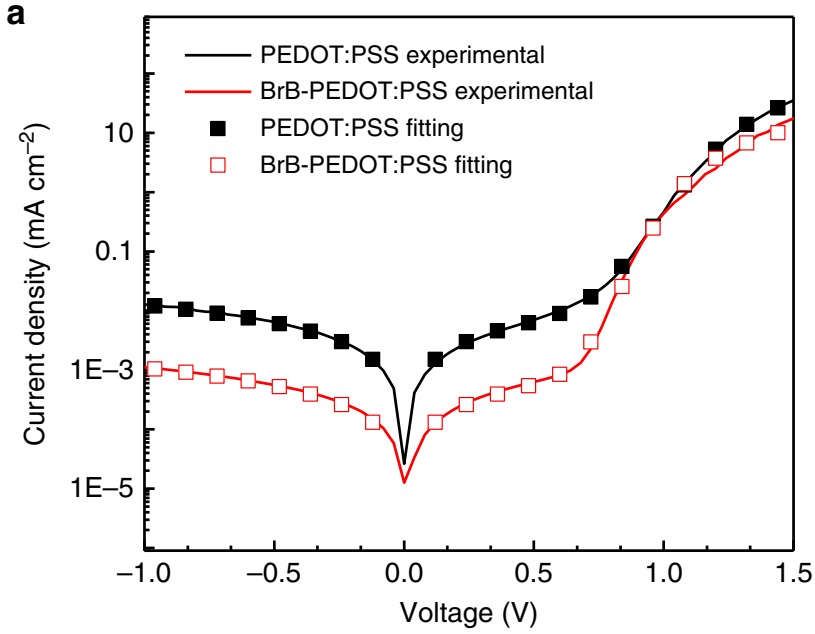

b

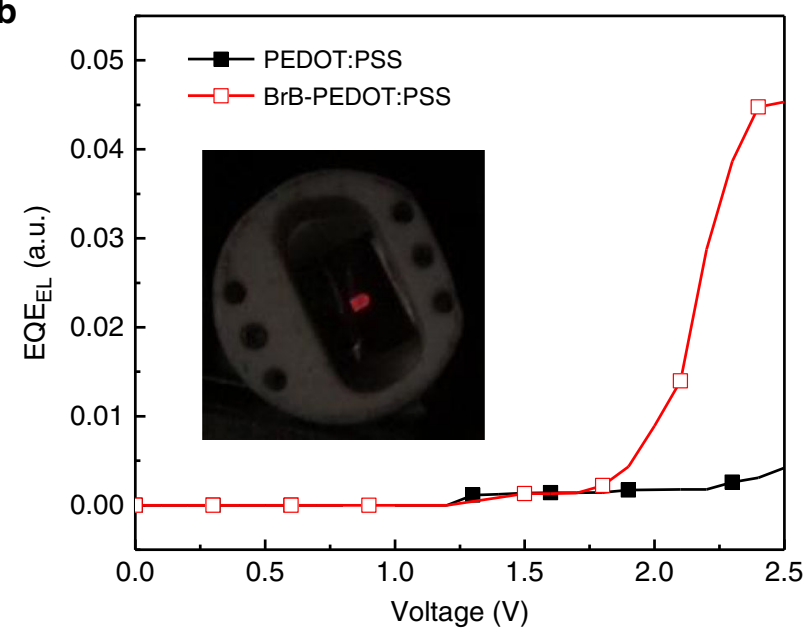

d

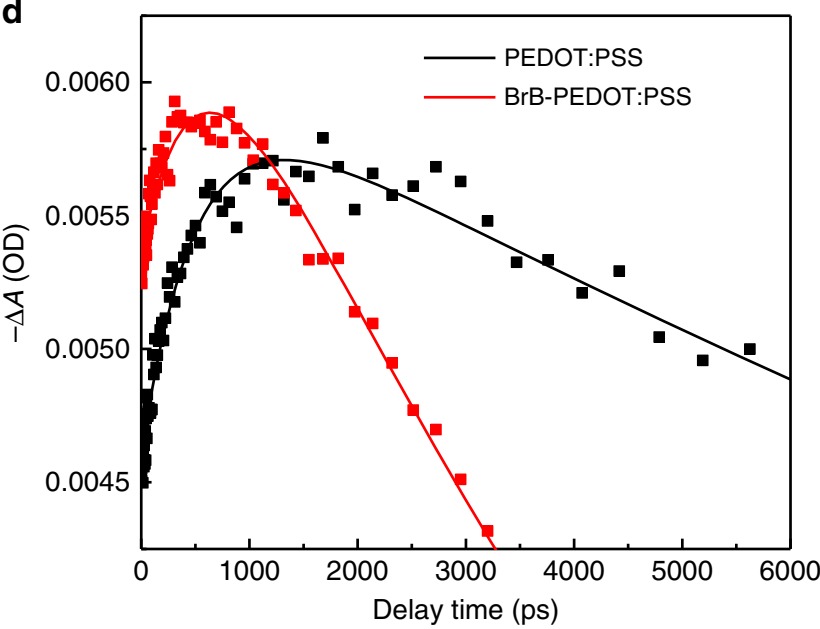

5 c

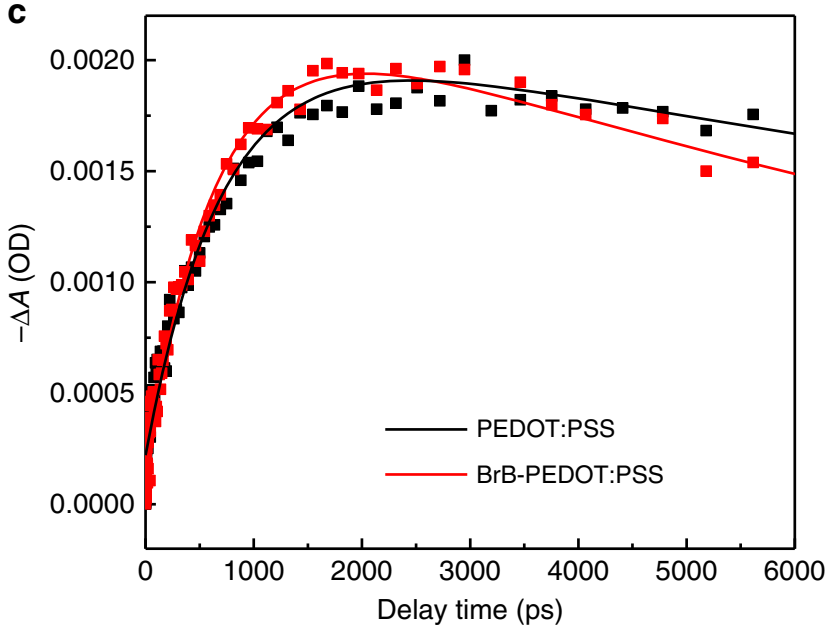


or without aperture. The measurement was performed in a $\mathrm{N}_{2}$-fillerd glove box at the temperature of $25^{\circ} \mathrm{C}$. Light intensity of the light source was calibrated with a silicon photodiode (Hamamatsu, S1133-01) which has been certified by National Renewable Energy Laboratory (NREL). The scan rate was $0.2 \mathrm{~V} \mathrm{~s}^{-1}$ and the dwell time was $0.1 \mathrm{~s}$

The external quantum efficiency (EQE) test was characterized using a $150 \mathrm{~W}$ xenon lamp (Oriel) filtered with a monochromator (Cornerstone 74004) as a monochromatic light source.

The EL measurement was performed on a home-built system. A Si photodiode (FU-G010, the area is $1 \mathrm{~cm}^{2}$ ) was placed on back of the device to record the EL intensity. A Keithley 2400 SourceMeter is used to apply the voltage and collect the current. The other 2400 SourceMeter was used to measure the current of the $\mathrm{Si}$ photodiode. The $\mathrm{EQE}_{\mathrm{EL}}$ was expected to be underestimated because angular dependence of emission and the detector sensitivity were not considered.

SEM images were conducted by a high-resolution field emission scanning electron microscope (FEI Nova Nano-SEM 450). The absorbance of Q-2D perovskite films was acquired by a UV-vis-NIR Spectrophotometer (UV-3600, Shimadzu). FT-IR experiments were performed on the VERTEX 70, Bruker. The PL measurements were performed using a fluorescence spectrometer (Edinburgh Instruments FLS920) with a $150 \mathrm{~W}$ Xe lamp as an excitation source at $450 \mathrm{~nm}$. Indepth XPS (AXIS-ULTRA DLD-600W) profiling was performed on the samples of glass/HTL/Q-2D, where the HTL is PEDOT:PSS or BrB-PEDOT:PSS. The samples were etched by $\mathrm{Ar}_{2}$. The $\mathrm{C}$ and $\mathrm{Pb}$ atom ratios were detected. The femtosecond $\mathrm{TA}$ setup is based on a regenerative amplified Ti:sapphire laser system from Coherent $(800 \mathrm{~nm}, 35 \mathrm{fs}, 6 \mathrm{~mJ}$ pulse, and $1 \mathrm{kHz}$ repetition rate), nonlinear frequency mixing techniques and the ultrafast TA spectrometer (Time-Tech Spectra, femtoTA-100). GIWAXS measurements were carried out with a Xeuss 2.0 SAXS/WAXS laboratory beamline using a $\mathrm{Cu}$ X-ray source $(8.05 \mathrm{keV}, 1.54 \AA$ ) and a Pilatus3R $300 \mathrm{~K}$ detector. The incidence angle is $0.3^{\circ}$.

The contact angle was measured via an optical video contact angle instrument (VCA Optima XE, AST) at room temperature and was determined after a water droplet placed on the sample. Zeta potential and the dynamic light scattering measurement were tested on a Malvern Zetasizer Naono ZS. Concentration for the two measurements of the samples is $1 \mathrm{mg} \mathrm{mL}^{-1}$

\section{Data availability}

The data that support the findings of this study are available from the corresponding author upon reasonable request. Source data of Figs. 1a, 1b, 1f, 2a, 2d, 4b-d, 5 and Supplementary Figures 2, 3, 5, 7-19 are provided as a Source Data file.

Received: 5 June 2018 Accepted: 31 January 2019

Published online: 20 February 2019

\section{References}

1. De Wolf, S. et al. Organometallic halide perovskites: sharp optical absorption edge and its relation to photovoltaic performance. J. Phys. Chem. Lett. 5 , 1035-1039 (2014).

2. Miyata, A. et al. Direct measurement of the exciton binding energy and effective masses for charge carriers in organic-inorganic tri-halide perovskites. Nat. Phys. 11, 582-594 (2015).

3. Dong, Q. et al. Solar cells. Electron-hole diffusion lengths $>175$ mum in solution-grown $\mathrm{CH} 3 \mathrm{NH} 3 \mathrm{PbI} 3$ single crystals. Science 347, 967-970 (2015).

4. Stranks, S. D. et al. Electron-hole diffusion lengths exceeding 1 micrometer in an organometal trihalide perovskite absorber. Science 342, 341-344 (2013).

5. Xing, G. et al. Long-range balanced electron- and hole-transport lengths in organic-inorganic CH3NH3PbI3. Science 342, 344-347 (2013).

6. Shi, D. et al. Solar cells. Low trap-state density and long carrier diffusion in organolead trihalide perovskite single crystals. Science $347,519-522$ (2015).

7. Yin, W. J., Shi, T. T. \& Yan, Y. F. Unique properties of halide perovskites as possible origins of the superior solar cell performance. Adv. Mater. 26, 4653-4658 (2014).

8. Weidman, M. C., Seitz, M., Stranks, S. D. \& Tisdale, W. A. Highly tunable colloidal perovskite nanoplatelets through variable cation, metal, and halide composition. ACS Nano 10, 7830-7839 (2016).

9. Noh, J. H., Im, S. H., Heo, J. H., Mandal, T. N. \& Seok, S. I. Chemical management for colorful, efficient, and stable inorganic-organic hybrid nanostructured solar cells. Nano Lett. 13, 1764-1769 (2013).

10. National Renewable Energy Laboratory. Best Research-Cell Efficiencies Chart. https://www.nrel.gov/pv/assets/images/efficiency-chart.png, 2018.

11. Niu, G. D., Guo, X. D. \& Wang, L. D. Review of recent progress in chemical stability of perovskite solar cells. J. Mater. Chem. A 3, 8970-8980 (2015).

12. Leijtens, T. et al. Stability of metal halide perovskite solar cells. Adv. Energy Mater. 5, 1500963 (2015).
13. Park, N. G., Gratzel, M., Miyasaka, T., Zhu, K. \& Emery, K. Towards stable and commercially available perovskite solar cells. Nat. Energy 1, 16152 (2016).

14. Zhao, X. \& Park, N. G. Stability issues on perovskite solar cells. Photonics 2, 1139 (2015)

15. Yang, J., Siempelkamp, B. D., Liu, D. \& Kelly, T. L. Investigation of $\mathrm{CH} 3 \mathrm{NH} 3 \mathrm{PbI} 3$ degradation rates and mechanisms in controlled humidity environments using in situ techniques. ACS Nano 9, 1955-1963 (2015).

16. Li, F. M. \& Liu, M. Z. Recent efficient strategies for improving the moisture stability of perovskite solar cells. J. Mater. Chem. A 5, 15447-15459 (2017).

17. Dou, L. et al. Atomically thin two-dimensional organic-inorganic hybrid perovskites. Science 349, 1518-1521 (2015).

18. Wang, N. N. et al. Perovskite light-emitting diodes based on solutionprocessed self-organized multiple quantum wells. Nat. Photonics 10, 699-705 (2016).

19. Wang, Z. et al. Efficient ambient-air-stable solar cells with 2D-3D heterostructured butylammonium-caesium-formamidinium lead halide perovskites. Nat. Energy 2, 17135 (2017).

20. Yan, J., Qiu, W., Wu, G., Heremans, P. \& Chen, H. Recent progress in 2D/ quasi-2D layered metal halide perovskites for solar cells. J. Mater. Chem. A 6, 11063-11077 (2018)

21. Cao, D. H., Stoumpos, C. C., Farha, O. K., Hupp, J. T. \& Kanatzidis, M. G. 2D homologous perovskites as light-absorbing materials for solar cell applications. J. Am. Chem. Soc. 137, 7843-7850 (2015).

22. Lermer, C. et al. Toward fluorinated spacers for MAPI-derived hybrid perovskites: synthesis, characterization, and phase transitions of (FC2H4NH3) 2PbCl4. Chem. Mater. 28, 6560-6566 (2016).

23. Smith, I. C., Hoke, E. T., Solis-Ibarra, D., McGehee, M. D. \& Karunadasa, H. I. A layered hybrid perovskite solar-cell absorber with enhanced moisture stability. Angew. Chem. Int. Ed. 53, 11232-11235 (2014).

24. Quan, L. N. et al. Ligand-stabilized reduced-dimensionality perovskites. J. Am Chem. Soc. 138, 2649-2655 (2016).

25. Stoumpos, C. C. et al. Ruddlesden-Popper hybrid lead iodide perovskite 2D homologous semiconductors. Chem. Mater. 28, 2852-2867 (2016).

26. Tsai, H. et al. Design principles for electronic charge transport in solutionprocessed vertically stacked $2 \mathrm{D}$ perovskite quantum wells. Nat. Commun. $\mathbf{9}$, 2130 (2018).

27. Chen, Y. et al. 2D Ruddlesden-Popper perovskites for optoelectronics. $A d v$. Mater. 30, 1703487 (2018).

28. Chen, Y. N. et al. Tailoring organic cation of $2 \mathrm{D}$ air-stable organometal halide perovskites for highly efficient planar solar cells. Adv. Energy Mater. 7, 1700162 (2017)

29. Tsai, H. H. et al. High-efficiency two-dimensional Ruddlesden-Popper perovskite solar cells. Nature 536, 312-317 (2016).

30. Soe, C. M. M. et al. Understanding film formation morphology and orientation in high member 2D Ruddlesden-Popper perovskites for highefficiency solar cells. Adv. Energy Mater. 8, 1700979 (2018).

31. Chen, A. Z. et al. Origin of vertical orientation in two-dimensional metal halide perovskites and its effect on photovoltaic performance. Nat. Commun. 9, 1336 (2018).

32. Zhang, X. et al. Phase transition control for high performance RuddlesdenPopper perovskite solar cells. Adv. Mater. 30, e1707166 (2018).

33. Zhang, X. Q. et al. Orientation regulation of phenylethylammonium cation based 2D Perovskite solar cell with efficiency higher than $11 \%$. Adv. Energy Mater. 8, 1702498 (2018)

34. Zhang, X. et al. Vertically oriented 2D layered perovskite solar cells with enhanced efficiency and good stability. Small 13, 1700611 (2017).

35. Qing, J. et al. Aligned and graded type-II Ruddlesden-Popper perovskite films for efficient solar cells. Adv. Energy Mater. 8, 1800185 (2018).

36. Zhou, N. et al. Exploration of crystallization kinetics in quasi two-dimensional perovskite and high performance solar cells. J. Am. Chem. Soc. 140, 459-465 (2018).

37. Zhang, X. et al. Stable high efficiency two-dimensional perovskite solar cells via cesium doping. Energy Environ. Sci. 10, 2095-2102 (2017).

38. Liu, J., Leng, J., Wu, K., Zhang, J. \& Jin, S. Observation of internal photoinduced electron and hole separation in hybrid two-dimentional perovskite films. J. Am. Chem. Soc. 139, 1432-1435 (2017).

39. Shang, Q. et al. Unveiling structurally engineered carrier dynamics in hybrid quasi-two-dimensional perovskite thin films toward controllable emission. J. Phys. Chem. Lett. 8, 4431-4438 (2017).

40. $\mathrm{Li}$, L. et al. Unraveling the growth of hierarchical quasi-2D/3D perovskite and carrier dynamics. J. Phys. Chem. Lett. 9, 1124-1132 (2018).

41. Zheng, K. B. et al. Inter-phase charge and energy transfer in RuddlesdenPopper 2D perovskites: critical role of the spacing cations. J. Mater. Chem. A 6, 6244-6250 (2018).

42. Guselnikova, O. A. et al. Tuning of PEDOT: PSS properties through covalent surface modification. J. Polym. Sci. Pol. Phys. 55, 378-387 (2017). 
43. Xiong, Z., Gu, T. \& Wang, X. Self-assembled multilayer films of sulfonated graphene and polystyrene-based diazonium salt as photo-cross-linkable supercapacitor electrodes. Langmuir 30, 522-532 (2014).

44. Yan, K. et al. Hybrid halide perovskite solar cell precursors: colloidal chemistry and coordination engineering behind device processing for high efficiency. J. Am. Chem. Soc. 137, 4460-4468 (2015).

45. Li, B., Li, M., Fei, C., Cao, G. \& Tian, J. Colloidal engineering for monolayer $\mathrm{CH} 3 \mathrm{NH} 3 \mathrm{PbI} 3$ films toward high performance perovskite solar cells. J. Mater. Chem. A 5, 24168-24177 (2017).

46. Johnston, N. \& Howell, L. G. Sedimentation equilibria of colloidal particles. Phys. Rev. 35, 274-282 (1930).

47. Lee, S. H. et al. Modified physico-chemical properties and supercapacitive performance via DMSO inducement to PEDOT: PSS active layer. Org. Electron. 15, 3423-3430 (2014).

48. Xia, Y. J., Sun, K., Chang, J. J. \& Ouyang, J. Y. Effects of organic inorganic hybrid perovskite materials on the electronic properties and morphology of poly(3,4-ethylenedioxythiophene):poly(styrenesulfonate) and the photovoltaic performance of planar perovskite solar cells. J. Mater. Chem. A 3, 15897-15904 (2015).

49. McMeekin, D. P. et al. Crystallization kinetics and morphology control of formamidinium-cesium mixed-cation lead mixed-halide perovskite via tunability of the colloidal precursor solution. Adv. Mater. 29, 1607039 (2017).

50. Noel, N. K. et al. Unveiling the influence of $\mathrm{pH}$ on the crystallization of hybrid perovskites, delivering low voltage loss photovoltaics. Joule 1, 328-343 (2017).

51. Nayak, P. K. et al. Mechanism for rapid growth of organic-inorganic halide perovskite crystals. Nat. Commun. 7, 13303 (2016).

52. $\mathrm{Bi}, \mathrm{C}$. et al. Non-wetting surface-driven high-aspect-ratio crystalline grain growth for efficient hybrid perovskite solar cells. Nat. Commun. 6, 7747 (2015).

53. You, J. et al. Moisture assisted perovskite film growth for high performance solar cells. Appl. Phys. Lett. 105, 183902 (2014).

54. Jiang, Y. et al. Enhancement of photovoltaic performance by utilizing readily accessible hole transporting layer of vanadium(V) oxide hydrate in a polymer-fullerene blend solar cell. ACS Appl. Mater. Interfaces 8, 11658-11666 (2016).

55. Bi, D. et al. Efficient luminescent solar cells based on tailored mixed-cation perovskites. Sci. Adv. 2, e1501170 (2016)

56. Saliba, M. et al. Incorporation of rubidium cations into perovskite solar cells improves photovoltaic performance. Science 354, 206-209 (2016).

57. Yao, J. et al. Quantifying losses in open-circuit voltage in solution-processable solar cells. Phys. Rev. Appl. 4, 014020 (2015).

58. Shao, Y. C., Yuan, Y. B. \& Huang, J. S. Correlation of energy disorder and open-circuit voltage in hybrid perovskite solar cells. Nat. Energy 1, 15001 (2016).

59. Leng, J., Liu, J., Zhang, J. \& Jin, S. Decoupling interfacial charge transfer from bulk diffusion unravels its intrinsic role for efficient charge extraction in perovskite solar cells. J. Phys. Chem. Lett. 7, 5056-5061 (2016).

60. Liu, T. et al. Nonreduction-active hole-transporting layers enhancing opencircuit voltage and efficiency of planar perovskite solar cells. ACS Appl. Mater. Interfaces 8, 33899-33906 (2016).

\section{Acknowledgements}

The work is supported by the National Natural Science Foundation of China (Grant No. 51773072, 21474035), by the Recruitment Program of Global Youth Experts, the HUST Innovation Research Fund (Grant No. 2016JCTD111, 2017KFKJXX012), the Science and Technology Program of Hubei Province (2017AHB040) and China Postdoctoral Science Foundation funded project (2016M602289). The authors would like to thank the Analytical and Testing Center of Huazhong University of Science and Technology for providing the facilities to conduct the characterization.

\section{Author contributions}

T.F.L., Y.Y.J. and Y.H.Z. conceived the idea. T.F.L., Y.Y.J, F.Q., L.H. and X.S.J. performed the solar cell fabrication, characterization, and optimization. L.L.S. contributed to the PL measurement. T.F.L. and S.X.X. measured the EQE and EL. M.C.Q. and X.H.L. performed the GIWAXS characterization. J.X.L. and S.Y.J. performed the TA measurement P.P. prepared the 4-bromobenzenediazonium tetrafluoroborate. F.Y.J. involved in the data analysis and discussion. Y.H.Z. directed this work. T.F.L. wrote the first draft of the manuscript. All the authors revised and approved the manuscript.

\section{Additional information}

Supplementary Information accompanies this paper at https://doi.org/10.1038/s41467 019-08843-5.

Competing interests: The authors declare no competing interests.

Reprints and permission information is available online at http://npg.nature.com/ reprintsandpermissions/

Journal peer review information: Nature Communications thanks Wanyi Nie and the other anonymous reviewers for their contribution to the peer review of this work.

Publisher's note: Springer Nature remains neutral with regard to jurisdictional claims in published maps and institutional affiliations.

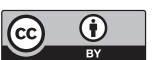

Open Access This article is licensed under a Creative Commons Attribution 4.0 International License, which permits use, sharing, adaptation, distribution and reproduction in any medium or format, as long as you give appropriate credit to the original author(s) and the source, provide a link to the Creative Commons license, and indicate if changes were made. The images or other third party material in this article are included in the article's Creative Commons license, unless indicated otherwise in a credit line to the material. If material is not included in the article's Creative Commons license and your intended use is not permitted by statutory regulation or exceeds the permitted use, you will need to obtain permission directly from the copyright holder. To view a copy of this license, visit http://creativecommons.org/ licenses/by/4.0/.

(c) The Author(s) 2019 